\title{
Design of Experimental Teaching Platform Based on Cloud Computing Virtualization Network
}

\author{
Jianmei Zhu \\ Wuhan Technology And Business University 430065,China
}

Keywords: computer information security, network attack and defense experiment, experimental teaching platform.

\begin{abstract}
A scheme based on penstock of construction of computer information security and network attack and defense experimental teaching platform is proposed. Meanwhile it is presented that its hardware topology architecture system technology architecture and the processes of platform management the technological principle of the virtual offensive and defensive carried out by students. There are 10 experimental modules in these platform curriculum nearly 100 experiment categories. Through the analysis of the teaching effect and contrast it shows that the platform can effectively improve the teaching quality.
\end{abstract}

\section{Introduction}

Cloud computing is a new generation of information resource sharing and application mode of IT resources to provide services to virtualization and resource pool, is the extension and development of network distributed computing, dynamic allocation of resources through virtualization technology, greatly improve the sharing of information resources and utilization. Cloud computing technology has been widely applied in various fields' resources of teaching and scientific research in complex information, computing technology integration of university resources using clouding order to improve the efficiency of teaching and experiment equipment and sharing provides practical solutions [1]. Therefore the research and development of university resources information platform based on cloud computing technology, achieving efficient resource utilization information of scientific research in Colleges and universities is of practical significance.

The campus network in the colleges has finished the coverage of the teaching axes, office area, training experimentation area, life service area and so on; digital communication equipment, servers and other resources in campus network, providing a strong guarantee for the college teachers and students in teaching, management, training, research, learning, life and other aspects [2]. But, with the expansion of college scale and the restriction of the traditional network technology, on the one hand, the college should continue to invest in expensive servers and other hardware devices; on the other hand, the information sharing degree is low in each department, and the information island is easy to form; and the low resource utilization at servers and other resources in different departments. Therefore, the campus network's management appears many problems, such as difficult, low efficiency and high cost.

\section{The function of experiment platform based on cloud technology and virtualization}

Support users through LAN access or internet access to the virtual simulation platform for information security experiments and attack and defense experiments. Virtual machine (VM), which is based on the virtual and the simulation and abstraction of the underlying device, generates the independent function. Virtualized technology is the core technology of cloud computing infrastructure-as-a-service (Iasi) [3], which solves the disadvantages of traditional campus network. 
In this paper, invested the current situation of the school network, analysis the existing problems of the campus network, the campus network virtualization technology in-depth researched, gave a upgrade from existing campus network virtualized technology to cloud computing infrastructure-as-a-service (IaaS). Selected Huawei CAS products of cloud computing virtualization management platform, realized the construction of a campus network computing virtualization, network virtualization, storage virtualization platform to build [4], deploy, I presided over a the college construction projects of carrying out the based cloud computing campus network virtualized platform (IaaS) [5]. Researched on the key technology of network cloud computing and virtualization Completed the work of campus network virtualization platform plane on. Nag construction, virtual resource deployment and management operation. Through the cloud management platform, in the light of creating, modify, tinplating, clone, migration operation, completed the added 6 servers' campus network virtualization resource deployment, the server utilization rate increased to more than $70 \%$, the cost decreased by 50\%,the virtual machine's creation and management, computing resource's virtualization deployment, created the Switch, configured the switch IRF 2, and also completed the work for adding 2 new cloud core switches and 6 cloud access switches network resource, made the network management more simple, created the shared memory and shared file system, deployed the added 3 new capacity IP storage devices SAN 72TB's structure storage resource virtualization, made the average disk utilization increased greatly. Realize the campus network virtualization resource management, cloud services and system testing work; In the light of the creation, modification, template, cloning, migration and other operations, compiled the VM management. Used EVB technology, completed VM traffic and edge management, for the organization and the administrator of the CAS, the creation of the user, complete the allocation of cloud service resources, etc.

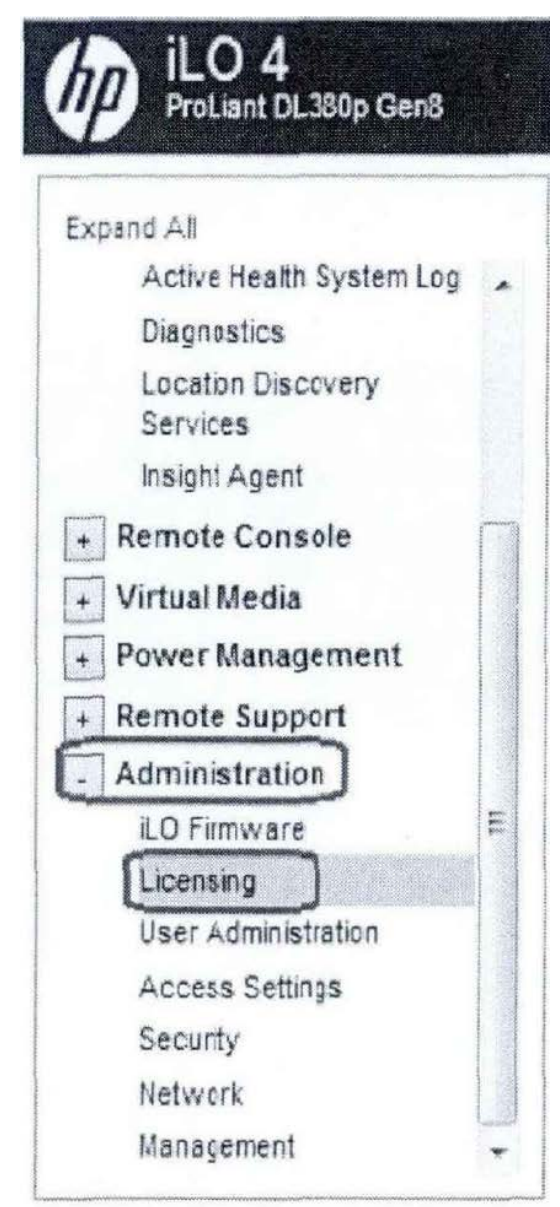

Fig. 1 IL0 4 management platform

In this paper, we completed the virtual deployment and application of the campus network. Through the infrastructure virtualization platform cloud deployment, effectively solved the problems such as low efficiency of server, Information Island and so on, improved the reliability and flexibility 
of the operation of the campus network equipment, made the campus network more intelligent and easy to use, had a very important significance to realize the education information negation in the Colleges.

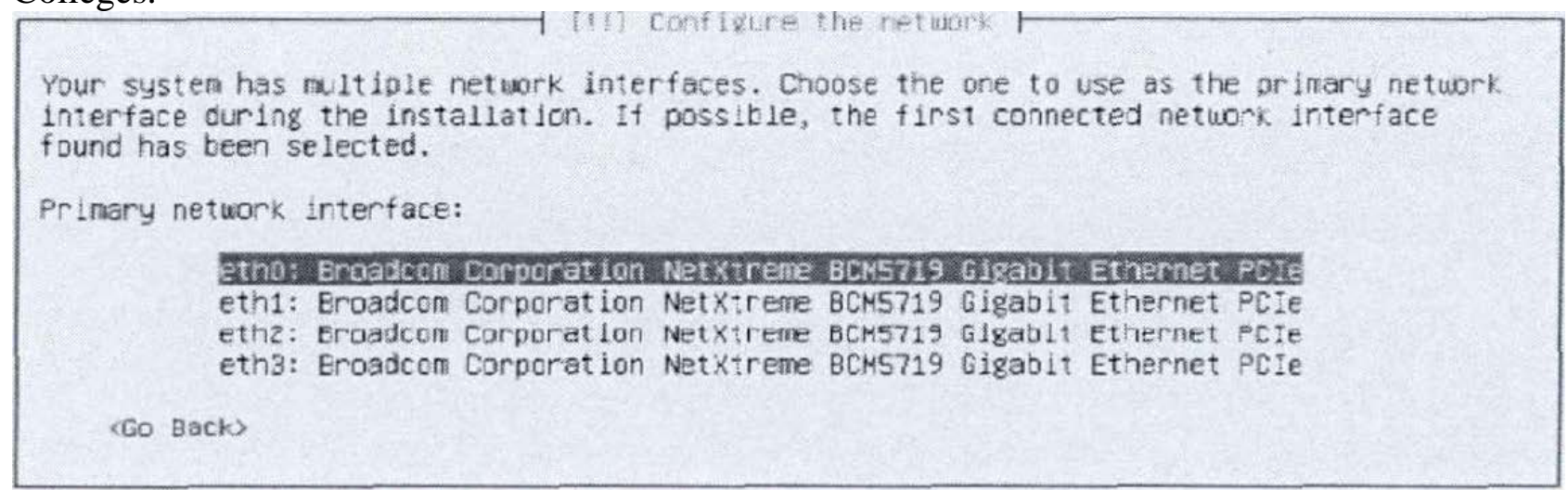

Fig. 2 The network configuration

\section{Construction plan of experiment platform}

The design of the experimental platform to provide self-service virtual experiment system of construction project, the use of virtual reality technology, a variety of media (such as audio, video, images and text) to assist the students' cognition and understanding of the virtual simulation experiment project content. According to the teaching requirements, students learn virtual simulation experiment knowledge online, and through network sharing platform of hardware and software resources, network security experiments. The platform can feed back the experimental process and the experimental data to the students for analysis and summary.

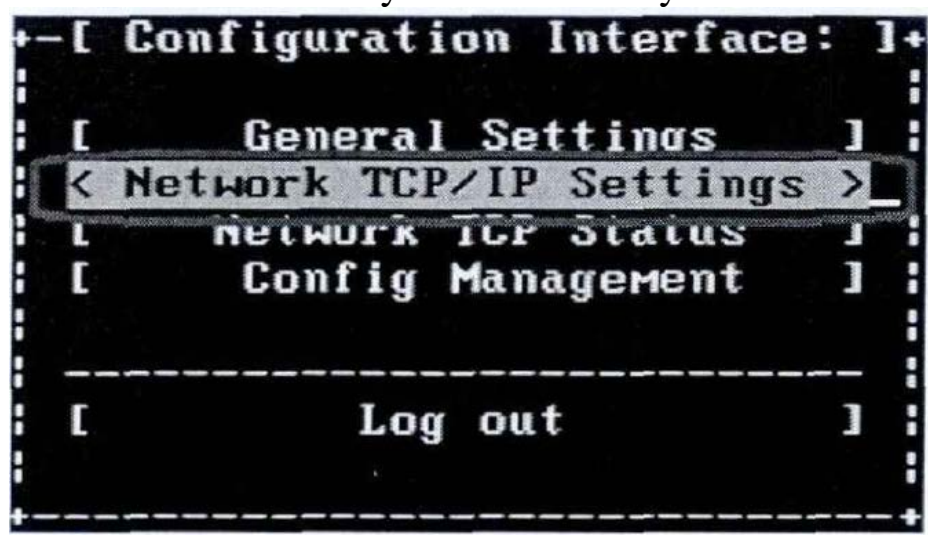

Fig. 3 Interface configuration interface

The thesis completed based on the cloud computing technology in teaching and research management platform, play a certain role in the practical application, and provide the basis for promoting the teaching resource management in Colleges and universities and the sharing of resources.

Control server and server nodes are installed Ubuntu operating system; which controls the server also installed Network module, the server node installed Nova and Swift, several other modules are also in the platform has been applied.

The experimental platform uses the control server to manage each server node, and establishes the virtual target machine and the virtual attack machine according to the mirror in the first network cloud storage and the second network cloud storage. Virtual machine and virtual attack machine can be connected through a virtual network, but also through the network security devices and network connection equipment to connect, in order to build a diverse network environment.

After the LAN user provides the correct user name and password, it is directly connected to the virtual simulation system. The Internet users establish the connection with the experimental platform through VPN. When LAN users and Internet users connect to the platform, you can use the disk space 
allocated by the administrator in advance for the first network cloud storage and the second network cloud storage.

Table 1 Cloud computing related parameters

\begin{tabular}{cccc}
\hline $\begin{array}{c}\text { Amazon } \\
\text { EC2 }\end{array}$ & Google APP & Microsoft Aure & Yahoo Hadoop \\
\hline IaaS & PaaS & PaaS & SaaS \\
Computer & Web & Web and non Web & Software \\
storage & application & & \\
OS on Xen & Application & OS through Fabric & Map Architecture \\
Hypervisor & container & & \\
\hline
\end{tabular}

\section{Summary}

This study based computing applications in teaching and research resources domestic in the study of cloud, and analyzes the current research object of existing research service for teaching and scientific research platform, discusses the advantages of teaching and research use cloud University in the existing teaching and research platform, based on the scientific research and teaching in Colleges and universities to design the architecture of cloud research by SQL Azure, provide cloud data service based on SQL Server, as the basis of data center construction supporting products, calculation theory and application model using cloud, construction of various types of resource fusion data structure model, constructing the scientific teaching platform based on cloud computing, including cloud computing resources integration and the overall deployment scheme based on design and construction; university teaching platform based on cloud computing, cloud computing, cloud university teaching platform framework architecture and overall solutions to do the design based on the design of function; university teaching platform based on cloud computing, explains the platform construction goal, development framework, and the educational administration teaching platform function module design; Study on the server network architecture educational platform of university teaching based on cloud computing and security The whole structure of the design; design plate student enrollment management system, the system was designed on the basis of the demand analysis; finally, based on the calculation of cloud implementation of constructing teaching platform is expounded and a part of source code.

\section{Acknowledgements}

Wuhan Technology And Business University Teaching Reform Project: Based on the "cloud classroom" practice teaching model research (project number: 2014Y11)

\section{References}

[1] Tan Y, Luo D, Wang J. CC-VIT: Virtualization Intrusion Tolerance Based on Cloud Computing (International Conference on Information Engineering and Computer Science. IEEE, 2010), p. 1-6. (In Chinese)

[2] Wei F, Xuefeng X. A Virtualization Resource Management Platform of Cloud Computing Based on the Xen (International Symposium on Information Science and Engineering. IEEE, 2013), p. 220-222.

[3] Wan Z. A Network Virtualization Approach in Many-core Processor Based Cloud Computing Environment (Third International Conference on Computational Intelligence, Communication Systems and Networks. IEEE, 2011), p. 304-307. 
[4] Xu C, Tian Q, Zhang H. A research of safety mechanism in cloud computing platform based on virtualization (International Conference on Computer Science \& Education. IEEE, 2012), p. 245-248.

[5] Zhang L, Liu M, Shi Z, et al. Research on Virtual Basic Laboratory and Experimental Teaching Resources Platform Based on Cloud Computing. (Physical Review), Vol. 271 (2017) No. 2, p. 549-554. 